\title{
Therapie der Herzinsuffizienz
}

\section{$\mathrm{AT}_{1}$-Antagonist bewährt sich auch im Praxisalltag}

VON M. BAUMHÄKEL, U. MÜLLER

\author{
Im Rahmen einer Praxisstudie erhiel- \\ ten Herzinsuffizienzpatienten den \\ $\mathrm{AT}_{1}$-Antagonisten Valsartan. Nach \\ zwölf Wochen zeigte sich eine Ver- \\ besserung der NYHA-Klassifikation \\ und der Lebensqualität.
}

- In der Therapie der chronischen Herzinsuffizienz spielt eine Hemmung des Renin-Angiotensin-Systems (RAS) eine zentrale Rolle. Trotz Gabe eines ACE-Hemmers können jedoch oft relevante Angiotensin-II-Plasmaspiegel nachgewiesen werden, die zu einer Progression der Herzinsuffizienz beitragen. Die zusätzliche Hemmung des RAS mithilfe eines $\mathrm{AT}_{1}$-Antagonisten erschien daher bezüglich der Sterblichkeit und Morbidität von Herzinsuffizienzpatienten von Vorteil zu sein. Bislang wurden die drei $\mathrm{AT}_{1}$-Antagonisten Valsartan, Losartan und Candesartan in randomisierten, kontrollierten Studien untersucht.

Mit dem Ziel, die Herzinsuffizienztherapie mit Valsartan unter Praxisbedingungen $\mathrm{zu}$ evaluieren, nahmen 1863 Patienten und 829 niedergelassene Ärzte an einer offenen, multizentrischen, prospektiven Untersuchung in Deutschland teil. Die Einschlusskriterien entsprachen der Zulassung von Valsartan bei chronischer Herzinsuffizienz. Die Dosierung lag im Ermessen des behandelnden Arztes

- Cordinate ${ }^{\circledR}$; Hersteller: AWD.pharma GmbH \& Co. KG, Radebeul

Die Studie wurde von AWD.pharma, Deutschland unterstützt. AWD.pharma war verantwortlich für die Organisation und das Datenmanagement der Studie. AWD.pharma hatte keinen Einfluss auf die Analyse sowie die Interpretation der Daten.

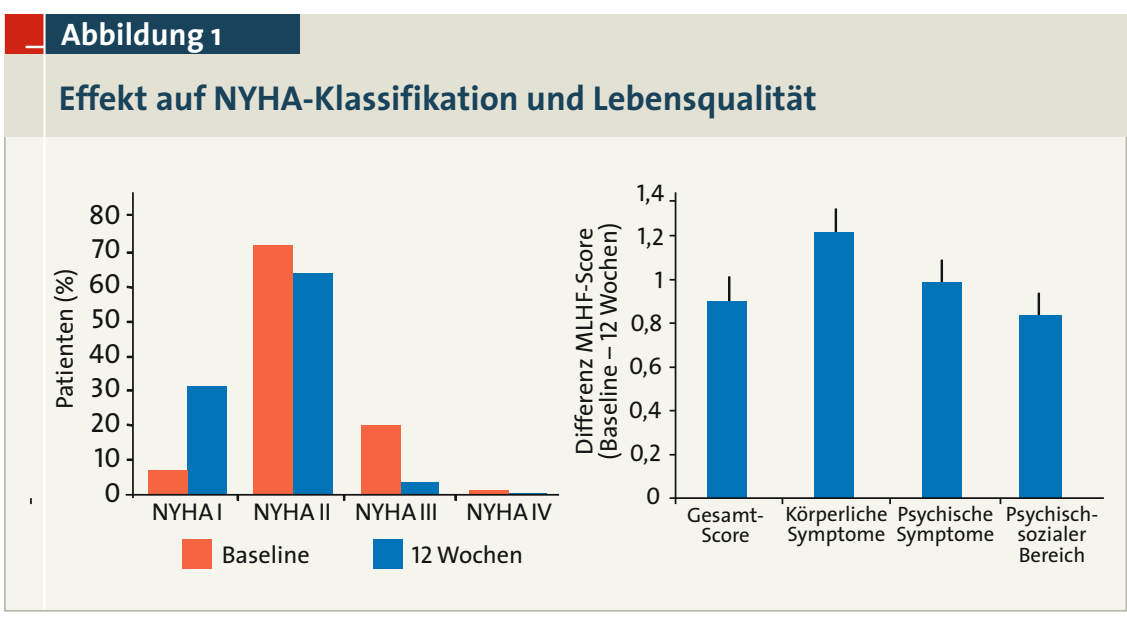

Abb. 1 Nach zwölfwöchiger Therapie mit Valsartan zeigte sich eine Verbesserung der NYHA-Klassifikation der Patienten ( $p<0,001$ Baseline vs. 12-Wochen-Therapie) und des Minnesota-Living-With-Heart-Failure-(MLHF-)Score.

und sollte gemäß den Empfehlungen der Fachinformation erfolgen. Die häufigste Begleitmedikation zu Beginn der Studie waren ACE-Hemmer $(73,6 \%)$, Diuretika $(67,7 \%)$ und Betablocker $(63,4 \%)$.

\section{Ergebnisse}

Nach zwölfwöchiger Therapie mit Valsartan verbesserten sich die NYHAKlassifikation und die Lebensqualität der Patienten signifikant. So erhöhte sich z. B. der Anteil der Patienten im NYHA-Stadium I von 7\% zu Beobachtungsbeginn auf $31 \%$ nach zwölf Wochen (Abb. 1). Die Verbesserung der Lebensqualität konnte sowohl im Gesamt-Score als auch in den Subdomänen des „Minnesota Living With Heart Failure-(MLHF-)Score" nachgewiesen werden (Abb. 1).

Den Allgemeinzustand beurteilten Ärzte und Patienten zum Abschluss der Therapie überwiegend als verbessert oder deutlich verbessert. Die Inzi- denz unerwünschter Arzneimittelwirkungen lag bei $0,2 \%$.

\section{Fazit}

Die Studie bestätigt die Resultate randomisierter, placebokontrollierter Studien bezüglich der Verbesserung der NYHA-Klassifikation und der Lebensqualität durch die leitliniengerechte Therapie der chronischen Herzinsuffizienz mit dem $\mathrm{AT}_{1}$-Antagonisten Valsartan auch unter ambulanter ärztlicher Versorgung.

Für die Verfasser:

Dr. med. Magnus Baumhäkel

Klinik für Innere Medizin III

Universitätsklinikum des Saarlandes

D-66421 Homburg/Saar

Koautorin:

Dr. Ulrike Müller, AWD.pharma GmbH \& Co. KG, Radebeul

Die ausführliche Langfassung der Studie sowie die Literatur finden Sie im Originalienergänzungsband der MMW Nr. 1/2008 und unter www.mmw.de. 